\title{
GASTRO-INTESTINAL PARASITES OF RED PANDA (Ailurus fulgens fulgens Cuvier, 1825) IN RARA NATIONAL PARK, MUGU, NEPAL
}

\author{
CHANDRA KAJI PAN SHRESTHA ${ }^{1}$ AND MAHENDRA MAHARJAN \\ ${ }^{1}$ Central Department of Zoology, Tribhuvan University \\ Kirtipur, Kathmandu, Nepal \\ mmaharjan@cdztu.edu.np
}

\begin{abstract}
A total of 22 faecal samples of Red panda were collected in the month of May/June 2011 and 21 faecal samples from the same site during the month of May/June 2012. All the samples were microscopically examined by faecal floatation and sedimentation techniques. Out of 43 faecal samples, 40 samples (93.02\%) were found positive for gastro-intestinal parasites. Altogether, 12 different species of gastro-intestinal parasites were recovered from Red panda. Among them, two species of protozoan parasites and 10 species of helminthes were observed. In protozoan parasites, Eimeria (67.44\%) was more prevalent as compared to Entamoeba (62.79\%). Among helminthes, Oxyuris sp. showed the highest prevalence rate followed by Toxoascaris sp. (48.84\%), Hookworm (44.19\%), Baylisascaris sp., Crenosoma sp. (34.88\%), Strongyloides sp., Moniezia sp. (18.60\%), Trichuris sp., etastrongylus sp. (4.65\%) and Angiostrongylus sp. (2.33\%). Only one species of cestoda i.e. Moniezia sp. was found but trematodes and acanthocephalans were not found in Red Panda of Rara National Park.
\end{abstract}

Keywords: prevalence, Eimeria, Entamoeba, Oxyuris, Angiostrongylus, Trichuris, Crenosoma

\section{INTRODUCTION}

Red panda (Ailurus fulgens fulgens Cuvier, 1825) is one of the poorly known small-bodied mammalian carnivores which belong to the order, Carnivora and the family Ailuridae. It is the only living species of the genus Ailurus and family Ailuridae (Flynn et al., 2000).

Globally, it is found in mountainous regions of India, China, Bhutan, Myanmar, Laos including Nepal (Glatston, 1994). It is currently distributed in Nepal, Bhutan, India, Myanmar and China, but was once widely distributed across Eurasia (Roberts \& Gittlemans, 1984).

It occurs in coniferous, deciduous, and mixed forests with dense understory (Choudhary, 2001; Pradhan et al., 2001; Roberts \& Gittlemans, 1984; Wei et al., 1999; Yonzon et al., 1991). Although, it is a carnivore, it has adapted to an almost completely herbivorous diet. It feeds upon bamboo leaves throughout the year and bamboo shoots in the spring (Wei et al., 1999).

Globally, it is estimated that there are fewer than 10,000 mature individuals, and populations continue to decline (Wang et al. 2008). It is categorized as vulnerable species (IUCN, 2015) and listed in Appendix I by CITES. In Nepal it is placed in the protected species by Government to conserve the species under National Parks and Wildlife Conservation Act (1973). 


\section{MATERIALS AND METHODS}

\section{Study area}

Rara National Park is located at the north-western high mountains ( $81^{\circ} 59^{\prime} 54^{\prime \prime}$ to $82^{\circ} 08^{\prime} 27^{\prime \prime} \mathrm{E}$, $29^{\circ} 26^{\prime} 28^{\prime \prime}$ to $29^{\circ} 33^{\prime} 11^{\prime \prime} \mathrm{N}$ ) of Nepal. It is the smallest national park with an area of approximately $106 \mathrm{~km}^{2}$ including the lake Rara which is situated at an altitude of $2990 \mathrm{~m}$. Most of the area of the park is located in Mugu District with a small portion in Jumla District of Karnali zone. Majority of the park landscape is dominated with conifers which is suitable habitat of about 51 species of different mammal species (DNPWC, 2012)

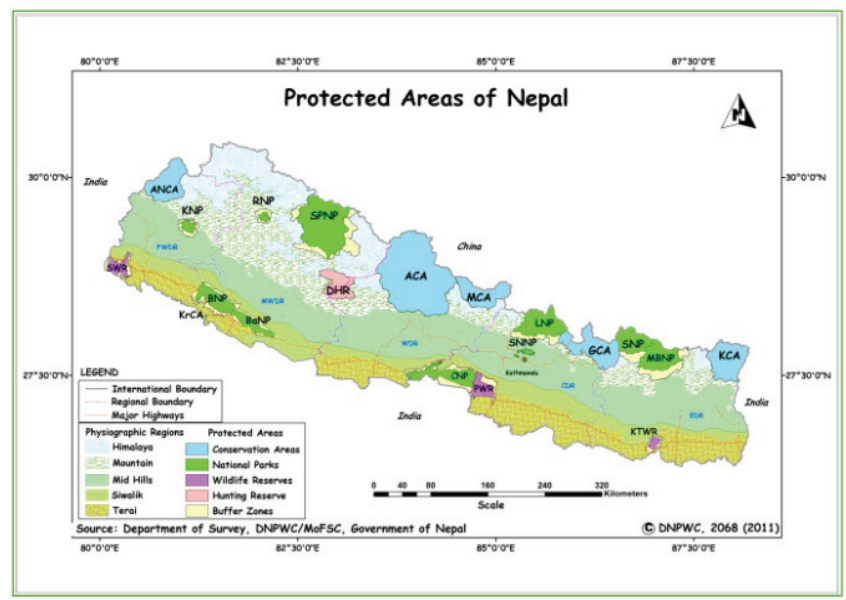

FIG. 1a. Map of Nepal showing the location of the Rara National Park.

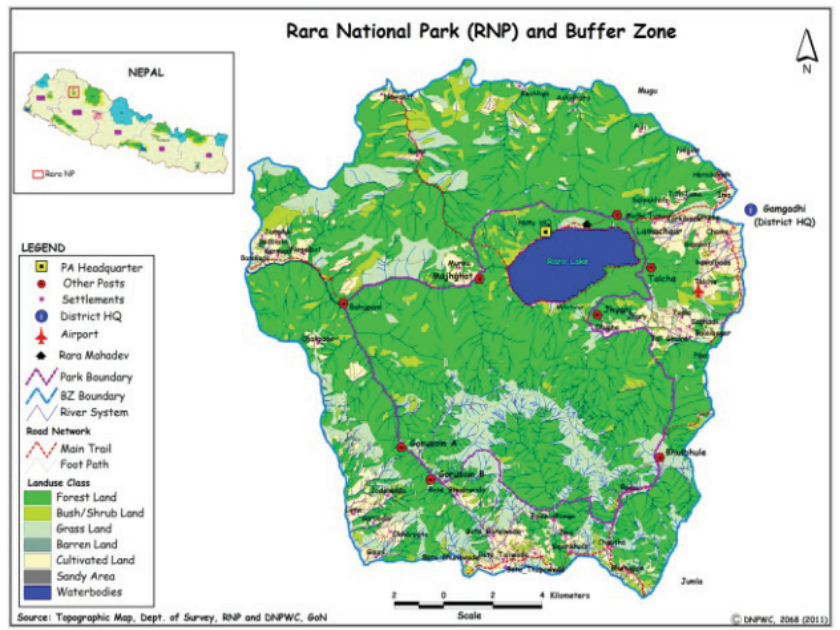

FIG. 1b. Map showing the study area Rara National Park. 


\section{Sample size and study design}

A total of 22 faecal samples of Red Panda were collected from the east-west facing slope of mountain surrounding the Rara Lake during the month of May/June 2011 and 21 faecal samples from the same site during the month of May/June 2012. The present study was designed to assess the gastrointestinal parasitic infection in Red panda (Ailurus fulgens) of Rara National Park by collecting faecal pellets using line transect method and analyze those using standard methods of eggs and oocyst detection.

\section{Sampling technique}

The site was divided into 12 line transects. First transect was marked at western end of mountain. Sample collection method was designed as shown in flow chart below. Briefly $30 \mathrm{~m}$ above the baseline (bank of Rara Lake) first plot was marked (T1P1). Each plot was of $10 \mathrm{~m} \times 10 \mathrm{~m}$ in size searched thoroughly for faecal pellets. Second plot was $100 \mathrm{~m}$ above the first plot and marked (T1P2) and so on. Faecal pellets were collected in the plot as well as in transect. Fresh faecal pellets were collected in sample collection plastic bags. Necessary information were marked clearly, such as faecal pellet collection date, altitude, transect and plot etc. Faecal pellets were preserved in Zipper plastic bags, brought to the Central Department of Zoology and filled with $2.5 \%$ potassium dichromate for preserving both helminthic eggs and protozoan cysts.

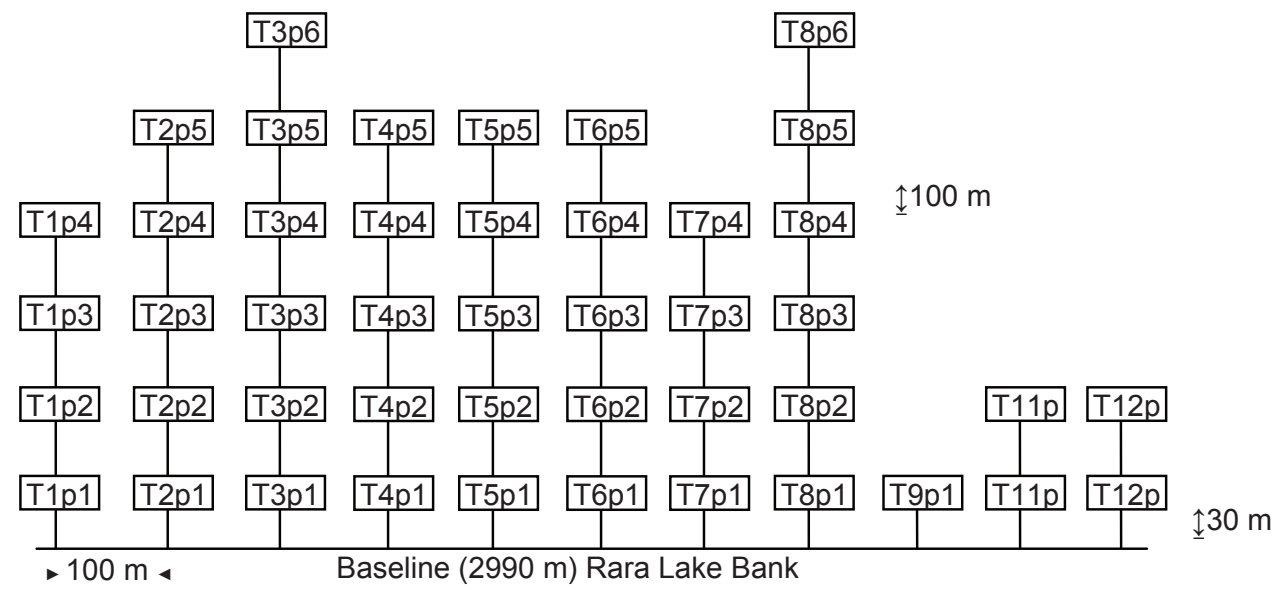

Flow Chart showing sample collection design.

\section{Examination of faecal samples}

Microscopic examination of collected faecal samples was carried out for the demonstration of cysts, eggs and larvae. Two concentration techniques (floatation and sedimentation techniques) and Stoll's Counting Method were followed. The faecal pellets were examined under microscope at the laboratory of Central Department of Zoology, Tibhuvan University, Kirtipur, Kathmandu. 


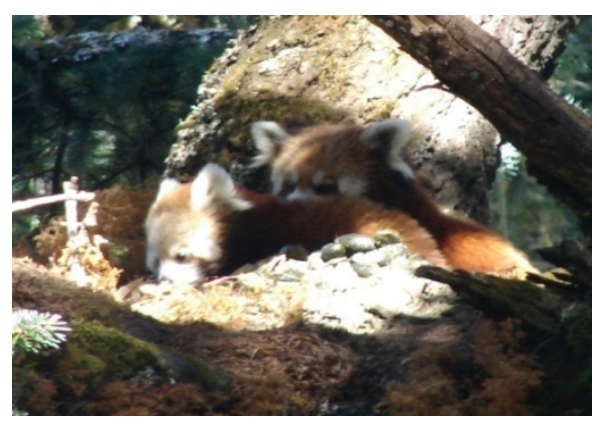

Red Panda in the field.

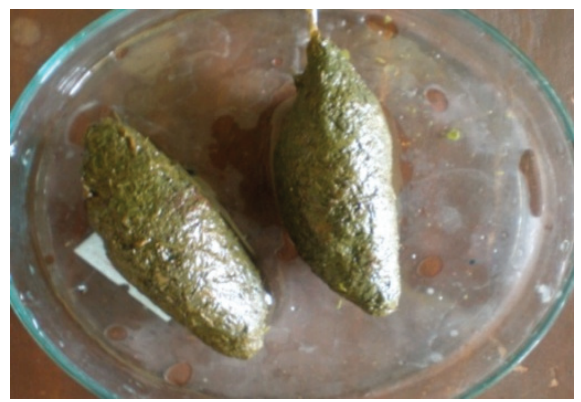

Faecal sample on Petridish.

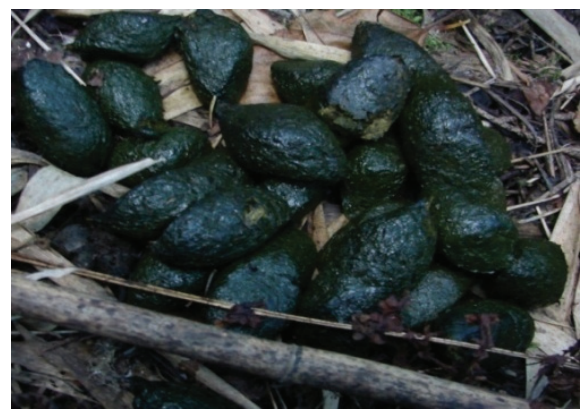

Faecal sample collection.

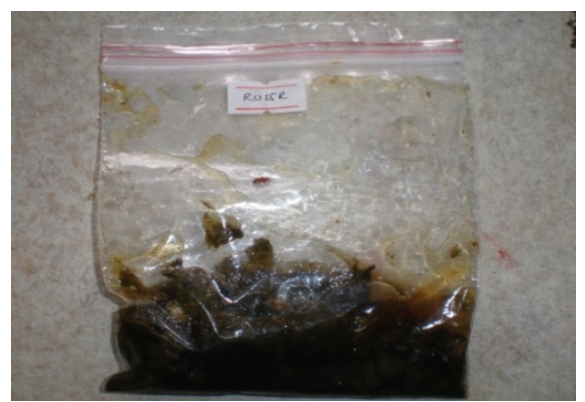

Faecal sample preserved in $2.5 \% \mathrm{~K} 2 \mathrm{Cr} 2 \mathrm{O} 7$.

\section{RESULTS AND DISCUSSION}

General prevalence of gastrointestinal parasites in Red panda

The prevalence of gastrointestinal parasites in Red panda was observed to be $93.00 \%$ (40/43). (fig. 2)

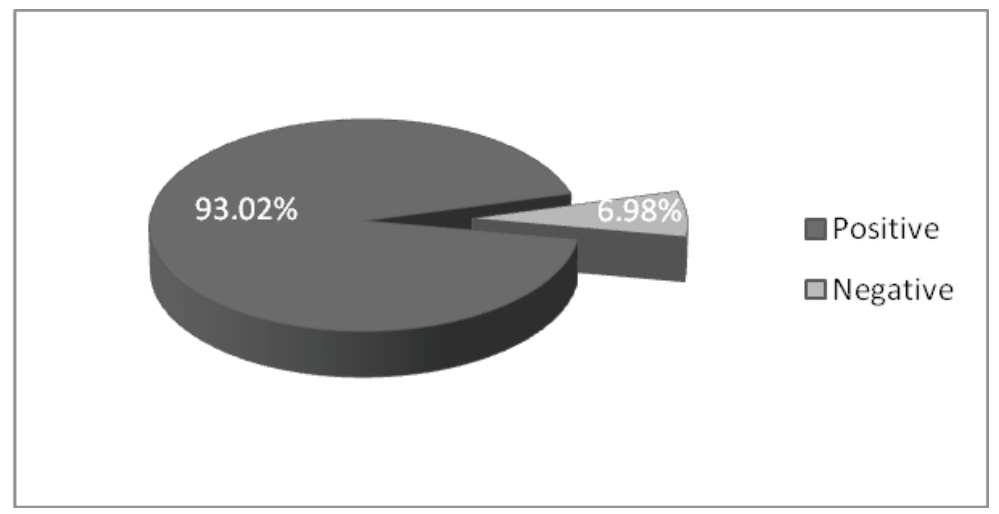

FIG. 2. Prevalence of gastrointestinal parasites in Red panda. 


\section{Class-wise prevalence of gastrointestinal parasites}

Among 43 samples examined, 33 samples (76.74\%), 37 samples ( $84.05 \%)$, and 8 samples $(18.60 \%)$ were found to be positive for protozoan, nematode and cestode infections respectively. Nematodes showed the highest prevalence rate with $84.05 \%$ and cestode showed the least prevalence rate with $18.60 \%$ (fig. 3 )

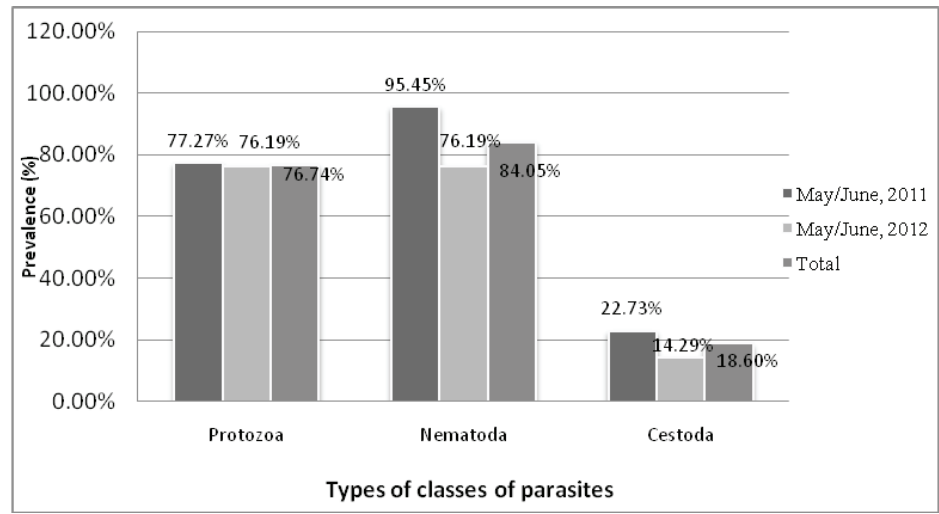

FIG. 3. Class-wise prevalence of gastrointestinal parasites in Red panda.

\section{Prevalence of protozoan parasites in Red panda}

Eimeria and Entamoeba species were identified by the examination of morphological shape, size and internal structure. Regarding coccidian parasites, two types of Eimeria were identified on the basis of presence or absence of micropyle. The higher prevalence rate was observed in the coccidian parasite, Eimeria as compared to Entamoeba, $67.44 \%$ against $62.79 \%$ (fig. 4 )

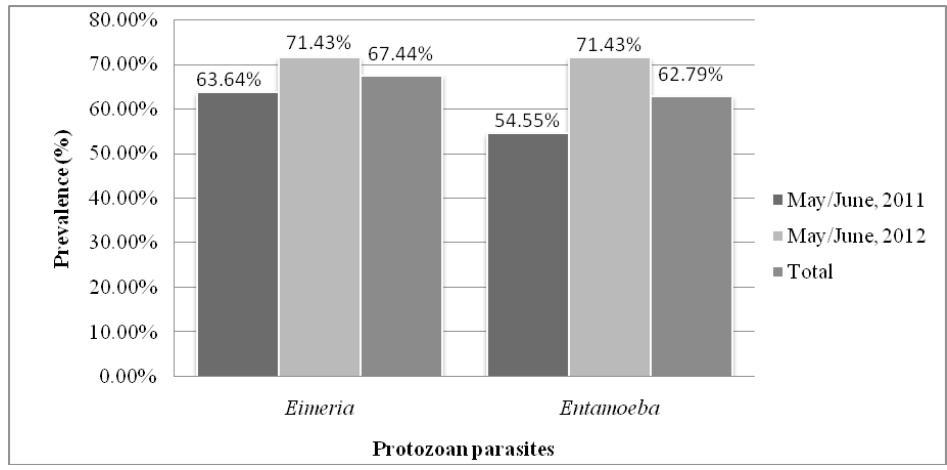

FIG. 4. Prevalence of protozoan parasites.

The size of the oocyst of Eimeria found in Red panda were greatly varied. The size of the oocyst ranges from $7.89 \mu \mathrm{m}-34.19 \mu \mathrm{m}$ in diameter and the size of the cyst of Entamoeba ranges from $21.04 \mu \mathrm{m}-28.93 \mu \mathrm{m}$ in diameter. 
Photographs showing different protozoan parasites at 400X magnification.
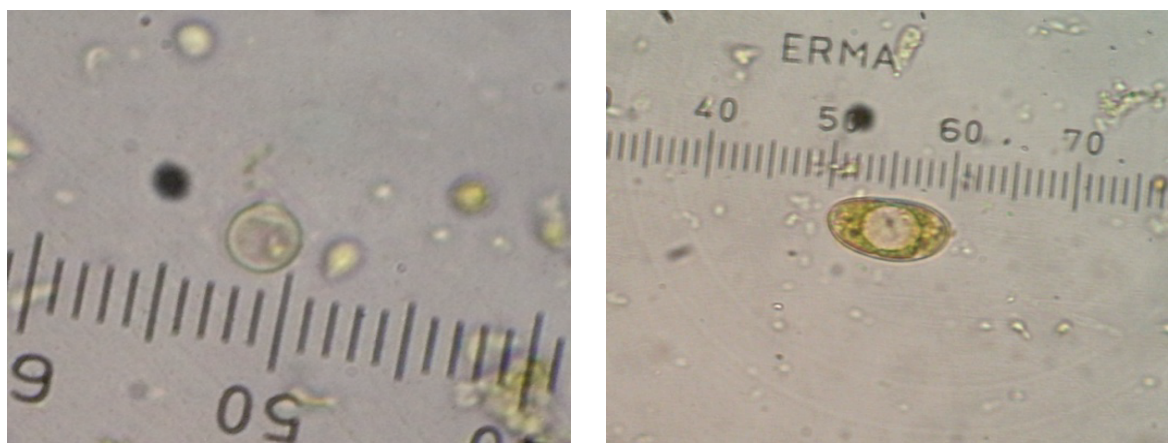

Plate 1. Oocyst of Eimeria with micropyle. Plate 2. Oocyst of Eimeria without micropyle.

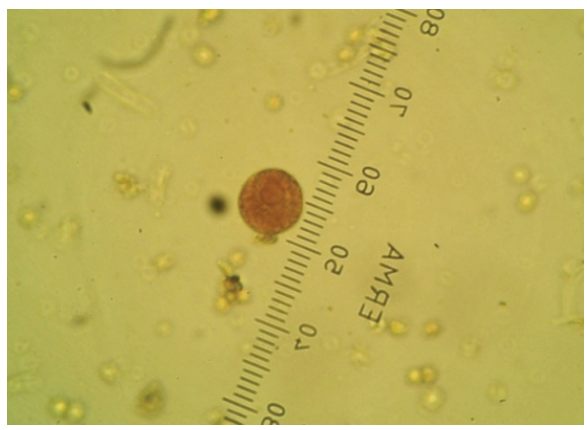

Plate 3. Cyst of Entamoeba.

\section{Prevalence of helminth parasites in Red panda}

Altogether 10 different types of helminthes were observed in Red panda, only one genera belonging to cestode and among 9 genera, 6 types of parasites were found to be intestinal worms (Hookworm, Toxoascaris sp., Baylisascaris sp., Strongyloides sp., Trichuris sp., Oxyuris $\mathrm{sp}$.) and 3 genera belong to lungworm nematode (Crenosoma sp., Angiostrongylus sp., Metastrongylus sp.) but trematodes were not observed during the study.

Out of 43 samples, the most prevalent helminth parasite was Oxyuris sp. (25 i.e.58.14\%) followed by Toxoascaris sp. (21 i.e. 48.84\%), Hookworm (19 i.e. 44.19\%), Baylisascaris sp. and Crenosoma sp. (15 i.e. $34.88 \%$ ), Strongyloides sp. and Moniezia sp. (8 each i.e. $18.60 \%$ ), Trichuris sp. and Metastrongylus sp. (2 each i.e. 4.65\%), Angiostrongylus sp. (1 i.e. 4.65\%) (table 1). 
TABLE 1. Prevalence of helminth parasites in Red panda.

\begin{tabular}{|c|c|c|c|}
\hline Types of parasites & $\begin{array}{c}\text { Positive samples from } \\
\mathbf{2 0 1 1}\end{array}$ & $\begin{array}{c}\text { Positive samples from } \\
\mathbf{2 0 1 2}\end{array}$ & Total \\
\hline Hookworm & $10(45.45 \%)$ & $9(42.86 \%)$ & $19(44.19 \%)$ \\
\hline Trichuris & $2(9.10 \%)$ & $0(0.00 \%)$ & $2(4.65 \%)$ \\
\hline Strongyloides & $6(27.27 \%)$ & $2(9.52 \%)$ & $8(18.60 \%)$ \\
\hline Baylisascaris & $8(36.36 \%)$ & $7(33.33 \%)$ & $15(34.88 \%)$ \\
\hline Toxoascaris & $12(54.45 \%)$ & $9(42.86 \%)$ & $21(48.84 \%)$ \\
\hline Moniezia & $5(22.73 \%)$ & $3(14.29 \%)$ & $8(18.60 \%)$ \\
\hline Oxyuris & $14(63.64 \%)$ & $11(52.38 \%)$ & $25(58.14 \%)$ \\
\hline Crenosoma & $6(27.27 \%)$ & $9(42.86 \%)$ & $15(34.88 \%)$ \\
\hline Angiostrongylus & $1(4.55 \%)$ & $0(0.00 \%)$ & $1(2.33 \%)$ \\
\hline Metastrongylus & $1(4.55 \%)$ & $1(4.76 \%)$ & $2(4.65 \%)$ \\
\hline
\end{tabular}

\section{Characteristics of helminths eggs}

Bean shaped and oval shaped eggs of Oxyuris sp. were observed ranging the size from $26.30 \mu-71.01 \mu \mathrm{m}$ in length. The eggs of Trichuris were barrel shaped with transparent mucous plug at either pole containing unsegmented embryo with average length $55.23 \mu \mathrm{m}-68.38 \mu \mathrm{m}$. Strongyloides eggs were ellipsoidal, thin walled, embryonated with $44.71 \mu \mathrm{m}$ in size. Eggs of Toxoascaris were observed ranging the size from $21.04 \mu \mathrm{m}-52.60 \mu \mathrm{m}$ while Baylisascaris eggs are typical ascarid eggs with thick, finely pitted shells; they are slightly smaller than Toxocara canis eggs. The eggs are oval in shape, with average, approximated dimensions of $80 \times 70 \mu \mathrm{m}$. Eggs of Baylisascaris were observed ranging from the size from $23.67 \mu-39.45 \mu \mathrm{m}$. Similarly, the eggs of Moniezia were triangular in shape containing a pyriform apparatus with the size ranging from $15.78 \mu \mathrm{m}-28.93 \mu \mathrm{m}$ in diameter.

\section{Concurrency of gastrointestinal parasites in Red panda}

In the present study, the multiple infections were observed in Red panda. Among 40 positive samples, 39 samples were found to have mixed infection with 2 to 9 species in each microscopic field. Among total of 43 samples, multiple and quintuple infections were found to be highest with $20.93 \%$ followed by quadruple $(18.60 \%)$, triple $(16.28 \%)$, double $(13.95 \%)$ and single infections (2.33\%) (table 2).

TABLE 2. Multiple infection of gastrointestinal parasites in Red panda.

\begin{tabular}{|l|l|l|l|}
\hline Concurrency & Occurrency (2011) & Occurrency (2012) & Total \\
\hline No Infection & $0(0.00 \%)$ & $3(13.64 \%)$ & $3(6.98 \%)$ \\
\hline Single Infection & $1(4.55 \%)$ & $0(0.00 \%)$ & $1(2.33 \%)$ \\
\hline Double Infection & $5(22.73 \%)$ & $1(4.76 \%)$ & $6(13.95 \%)$ \\
\hline Triple Infection & $3(13.64 \%)$ & $4(19.05 \%)$ & $7(16.28 \%)$ \\
\hline Quadruple Infection & $4(18.18 \%)$ & $4(19.05 \%)$ & $8(18.60 \%)$ \\
\hline Quintuple Infection & $5(22.73 \%)$ & $4(19.05 \%)$ & $9(20.93 \%)$ \\
\hline Multiple Infection & $4(18.18 \%)$ & $5(23.81 \%)$ & $9(20.93 \%)$ \\
\hline
\end{tabular}




\section{Intensity of infection of gastrointestinal parasites in Red panda}

Intensity of parasitic infection has been calculated based upon the number of eggs/oocyst and larvae found per field. Among protozoans, the high intensity of lightly infected cases was observed due to Eimeria sp. with $9(20.93 \%)$ samples and mildly infected cases was due to Eimeria sp. with $14(32.56 \%)$ samples while high intensity of moderately infected cases and heavily infected cases were due to Entamoeba sp. with $5(11.63 \%)$ samples and $3(6.98 \%)$ samples respectively. Among helminthes, the high intensity of lightly infected cases and mildly infected cases were observed due to Oxyuris sp. with 11 (25.58\%) samples and high intensity of moderately infected cases was due to Toxoascaris sp. with $4(9.30 \%)$ samples. Similarly, high intensity of heavily infected cases was due to the Baylisascaris spp. and Trichuris spp. with $2(4.65 \%)$ samples followed by Oxyuris sp. with $1(2.33 \%)$ sample (table 3$)$.

TABLE 3. Overall intensity of infection of gastrointestinal parasites in Red panda.

\begin{tabular}{|l|l|l|c|c|c|c|}
\hline SN & Class & Name of species & + & ++ & +++ & ++++ \\
\hline 1 & Protozoa & Eimeria spp. & 9 & 14 & 4 & 2 \\
\hline 2 & & Entamoeba spp. & 7 & 12 & 5 & 3 \\
\hline 3 & Nematoda & Hookworm spp. & 10 & 7 & 2 & - \\
\hline 4 & & Strongyloides spp. & 4 & 3 & 1 & - \\
\hline 5 & & Toxoascaris spp. & 7 & 10 & 4 & - \\
\hline 6 & & Baylisascaris spp. & 4 & 6 & 3 & 2 \\
\hline 7 & & Oxyuris spp. & 11 & 11 & 2 & 1 \\
\hline 8 & & Crenosoma sp. & 5 & 8 & 2 & - \\
\hline 9 & & Angiostrongylus sp. & - & 1 & - & - \\
\hline 10 & & Metastrongylus sp. & - & 2 & - & - \\
\hline 11 & & Trichuris spp. & - & - & - & 2 \\
\hline 12 & Cestoda & Moniezia spp. & 3 & 3 & 2 & - \\
\hline
\end{tabular}

$+=$ less than 2 ova per field i.e. light infection

$++=2-4$ ova per field i.e. mild infection

$+++=4-6$ ova per field i.e. moderate infection

$++++$

$=6$ or more ova per field i.e. heavy infection 
Photographs showing different protozoan parasites at 400X magnification.

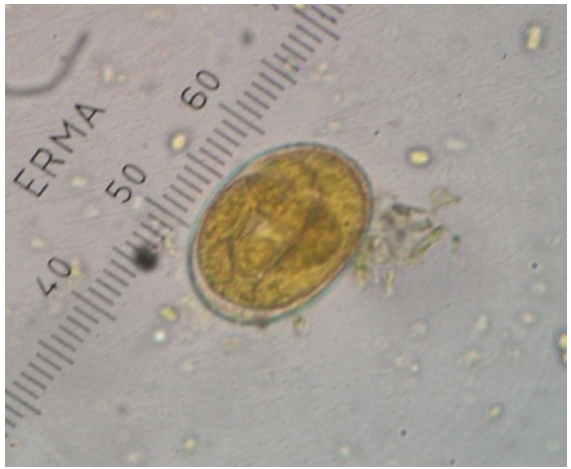

Plate 4. Egg of Strongyloides sp.

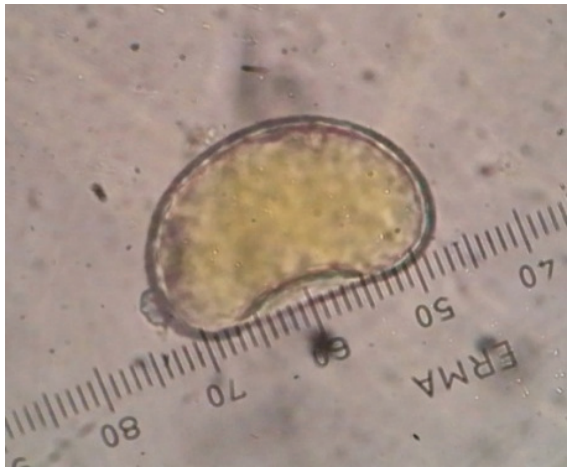

Plate 6. Egg of Oxyuris sp.

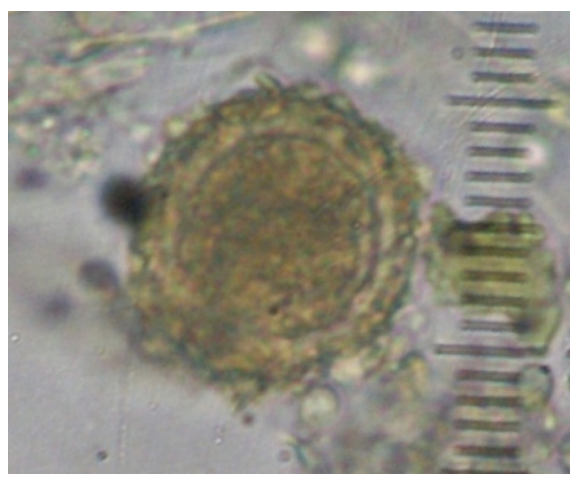

Plate 8. Egg of Baylisascaris sp.

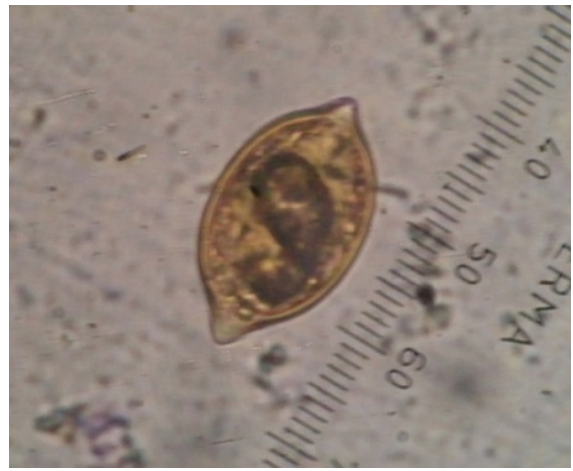

Plate 5. Egg of Trichuris sp.

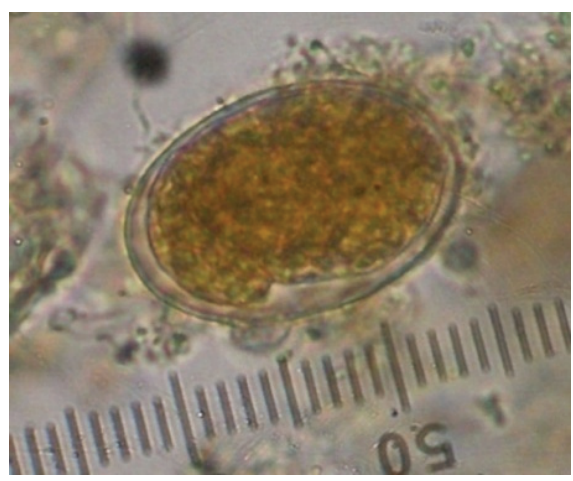

Plate 7. Egg of Hookworm.

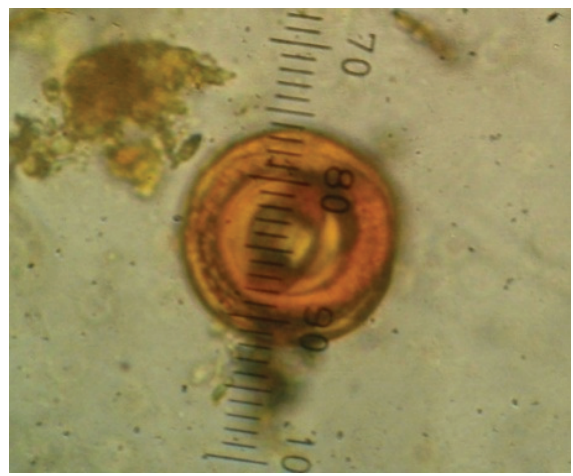

Plate 9. Egg of Toxoascaris sp. 


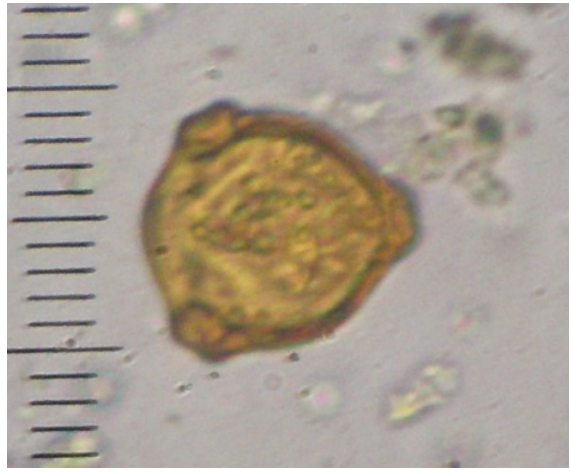

Plate 10. Egg of Moniezia sp.

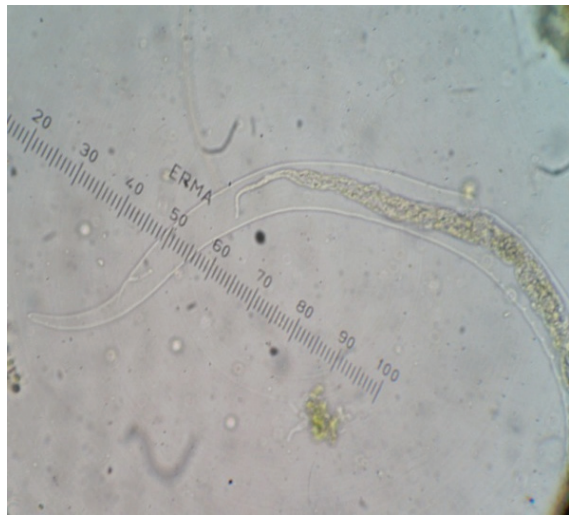

Plate 12. Larva of Crenosoma sp.

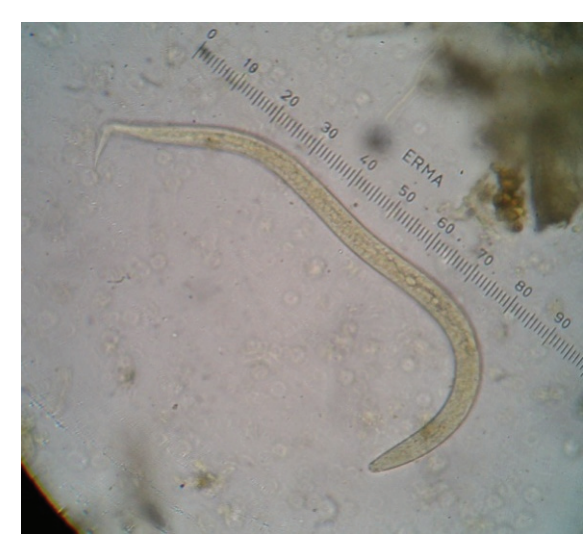

Plate 14. Larva of Metastrongylus sp.

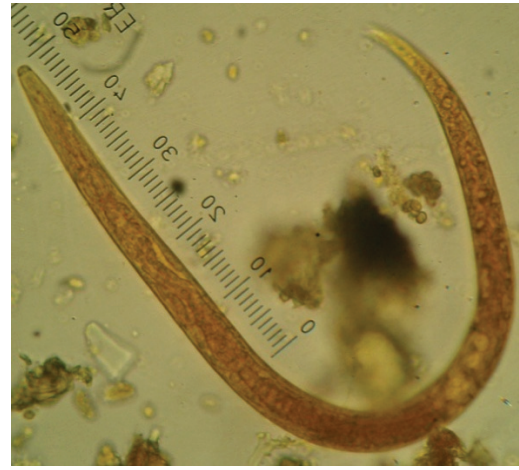

Plate 11. Larva of Strongyloides sp.

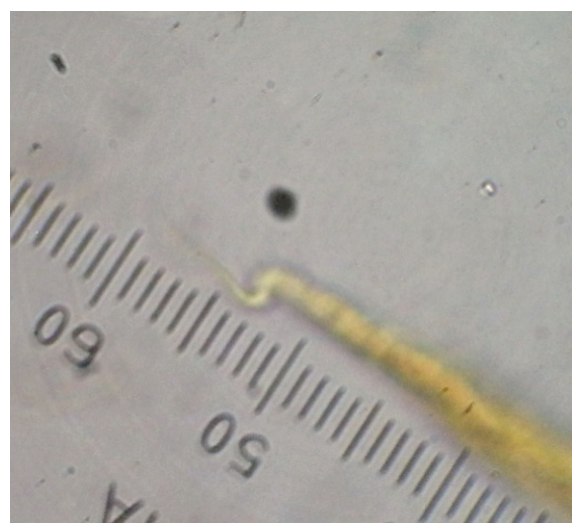

Plate 13. Larva of Angiostrongylus sp. 
No organism is free of parasites and parasites are cosmopolitan in distribution. Gastrointestinal parasites are found in human, wildlife and domestic animals. Wild animals harbour a wide range of parasites as definitive and reservoir hosts. This study attempts to document parasitic fauna of Red panda (Ailurus fulgens fulgens) in Rara National Park, Nepal. In the present study, altogether 12 different genera of parasites were reported from 40 out of 43 faecal samples of Red pandas. This high rate of gastrointestinal parasites may be due to different factors such as feeding behaviour, habitat, defecating openly on the ground and on trees, etc. Besides, parasites can also be easily transmitted through mechanical vectors like flies, rats, birds, lizards, cockroaches and beetles from one faecal matter to another. The pandas can also be infected by consuming the contaminated water and vegetation with infective stages of the parasites such as cysts, eggs, oocysts and larval forms. This may be the reason why $93.02 \%$ samples were found to be infected with at least one type of gastrointestinal parasites. Out of 43 samples, 33 samples $(76.74 \%), 37$ samples $(84.05 \%)$, and 8 samples $(18.60 \%)$ were found to be positive for protozoan, nematode and cestode infections respectively. In general, the parasites having direct life cycle have high prevalence rate e.g. coccidian and gastrointestinal nematodes while the parasites having indirect life cycle such as cestodes e.g. Moniezia sp. and trematodes have low prevalence rate. This is why nematodes were highly prevalent $(84.05 \%)$ and cestode was least prevalent (18.60\%). Both the protozoan parasites, Eimeria sp. and Entamoeba sp. have also high prevalence rate of $67.44 \%$ and $62.79 \%$ respectively. Thus, the prevalence rate of the coccidian parasite, Eimeria was found to be slightly higher as compared to Entamoeba. A recent study, from 23 faecal samples of Red panda in Nepal also revealed that the occurrence rate of protozoan parasites such as coccidians, Cryptosporidium and Cyclospora was $100 \%$ following nematodes $52.2 \%$, unidentified species trematodes $13.0 \%$ and unidentified species of cestodes 4.3\% (Lama et al., 2015). This showed that Eimeria and Entamoeba were commonly found in Red panda and had not been reported in any previous studies. This is the first case to report the Eimeria and Entamoeba in Red panda. A study carried out in Padmaja Naidu Himalayan Zoological Park, Darjeeling, India showed infection by Trichomonas sp. as protozoan parasite (Pradhan et al., 2011).

Altogether 10 different types of helminthes were reported in Red panda from Rara National Park. Helminthes included only one genus of cestode and 9 different types of nematodes among which 6 types of nematodes belonged to intestinal worms and 3 genera belonged to the lungworm nematodes. But trematodes and acanthocephalans were not observed during the study. However, the trematode Ogmocotyle ailuri was previously described from the Red panda, Ailurus fulgens, at a zoo in the United States (Price, 1954; Price, 1960). This trematode was also reported from the small intestine of Taiwanese monkeys, Macaca cyclopis (Yoshimura et al., 1969) and in the small intestine of Japanese monkey, Macaca fuscata, captured in Sendai City, Miyagi Prefecture, Japan (Iwaki et al., 2012). This shows that Ogmocotyle ailuri has a wide range of hosts. A study carried out in PNHZP, Darjeeling, India showed a trematode, Schistosoma sp. was reported in Red Panda (Pradhan et al., 2011) and similarly an unidentified trematode species were also recently reported in Red panda in Nepal (Lama et al., 2015). Only one genus, Moniezia was found in this study and the prevalence was found to be $18.60 \%$. This is the first record of the genus Moniezia from Red panda in the global context. However, an unidentified cestode species were recently reported in Red panda in Nepal (Lama et al., 2015). 
In this study, the prevalence of Oxyuris sp. was found to be the highest (58.14\%) among nematodes followed by Toxoascaris (48.84\%), Hookworm (44.19\%), Baylisascaris $(34.88 \%)$, Crenosoma (34.88\%), Strongyloides (18.60\%), Trichuris (4.65\%) Metastrongylus (4.65\%) and Angiostrongylus (2.33\%). Among these, Oxyuris spp., Toxoascaris sp., Hookworms, Strongyloides sp., and Metastrongylus sp. are reported for the first time in the global context. But Crenosoma spp. and Angiostrongylus spp. had been reported from Red Panda in the previous studies from different places (Grondahl et al., 2005; Patterson-Kane et al., 2009; Bertelsen et al., 2010) and more recently Angiostrongylus spp. was reported in 2 of 23 faecal samples, Trichuris in 6 of 23 faecal samples and Baylisascaris in 3 of 23 samples in Red panda in Nepal (Lama et al., 2015). Baylisascaris spp., a parasite favouring humans, utilizes more than 100 species of birds and mammals as hosts. These nematodes are pathologically very important because they appear as ocular, visceral and neural larvae migrans, resulting in blindness, loss of muscle control, hapatomegaly and coma. Trichuris spp. was not common in the samples but the parasite has been reported from humans, pets, livestock and wild animals such as foxes and dogs (Traversa, 2011).

Among total of 43 samples, multiple infections and quintuple infections were found to be highest with $20.93 \%$ followed by quadruple $(18.60 \%)$, triple $(16.28 \%)$, double $(13.95 \%)$ and single infection $(2.33 \%)$. Single infection was observed only in one faecal sample while double infection in 6 samples. The intensity of infection of gastrointestinal parasites was studied in this study in which both protozoans were found to be heavily infected. Similarly, among helminthes, Baylisascaris, Oxyuris and Trichuris were found to be heavily infected. Light infection may be asymptomatic but heavy infections cause diseases.

\section{ACKNOWLEDGEMENTS}

We would like to thank Mr. Bir Bahadur Buda, Game Scout of Rara National Park, Mr. Hari Prasad Sharma, Lecturer, CDZ as well as grantee of Rufford Small Grants Foundation for their respective support to make this possible.

\section{REFERENCES}

BERTELSEN, M F; MEYLAND-SMITH, F; WILLESEN, J L; JEFFERIES, R; MORGAN, E R; MONRAD, J (2010) Diversity and prevalence of metastrongyloid nematodes infecting the red panda (Ailurus fulgens) in European zoos. Veterinary Parasitology 172: 299-304.

CHAUDHARY, A (2001) An overview of the status and conservation of the Red panda Ailurus fulgens in India, with reference to its global status. Oryx 35(3): 250-259.

CUVIER, F (1825) Histoire naturelle Des Mamniferes avec.des Figures Originales.calories. Desinees d' Apris des Vicants: 1-3.

FLYNN, J J; NEDBAL, MA; DRAGOO, J W; HONEYCUTT, R L. (2000) "Whence the Red panda?". Molecular Phylogenetics and Evolution 17 (2): 190-199.

GLATSTON, AR (compiler) (1994) Status survey and conservation action plan for procyonids and Ailurids: the Red Panda, Olingos, Coatis, Raccoons and their Relatives. International Union for Conservation of Nature, Gland, Switzerland.

GRONDAHL, C; MONRAD, J; DIETZ, H H; JENSEN, H E; JOHANSEN, M V; KAPEL, C (2005) 
Angiostrongylosis in red panda (Ailurus fulgens fulgens). Proceedings of International Sympossium od Disease in Zoo and Wild animals 42: 117-118.

IWAKI, T; OKADA, T; SEKI, K; IZAWA, K; SAKURAI, F (2012) Ogmocotyle ailuri (Price, 1954) (Digenea: Notocotylidae) found in the Japanese monkey, Macaca fuscata. Journal of Veterinary Medical Science 74(9): 1211-1212.

JENSEN, H E; DIETZ, H H; GRONDAHL, C; KAPEL, C; MONRAD, J (2005) The pathology associated with Angiostrongylus vasorum infection in the red panda (Ailurus fulgens fulgens). Proceedings of the $23^{\text {rd }}$ Meeting of the European Society of Veterinary Pathology, The European Society of Veterinary Pathology 70-71.

LAMA, S T; LAMA, R P; REGMI, G R; GHIMIRE, T R (2015) Prevalence of intestinal parasitic infections in free-ranging Red panda Ailurus fulgens Cuvier, 1825 (Mammalia: Carnivora: Ailuridae) in Nepal. Journal of Threatened Taxa 7(8): 7460-7464.

PATTERSON-KANE, J C; GIBBONS, L M; JEFFERIES, R; MORGAN, E R; WENZLOW, N; REDROBE, S P (2009) Pneumonia from Angiostrongylus vasorum Infection in a Red panda (Ailurus fulgens fulgens) Journal of Veterinary Diagnostic Investigation 21(2): 270-273.

PRADHAN, S; SAHA, G K; KHAN, JA (2001) Ecology of the red panda (Ailurus fulgens) in the Singhalila National Park, Darjeeling, India. Biological Conservation 98: 11-18.

PRADHAN, S; SHARMA, D; SUBBA, B; CHETTRI, V (2011) Preliminary investigation on the parasites of mammals at Padmaja Naidu Himalayan Zoological Park, Darjeeling. Zoo's PRINT 26 (8): 11-13.

PRICE, E W (1954) A new trematode from the lesser panda, Ailurus fulgens. Journal of Parasitology 40 (5, sect. 2, suppl.): 38-39.

PRICE, E W (1960) A note on Ogmocotyle ailuri (Price, 1954) (Trematoda: Notocotylidae). Proceeding of the Helminthological Society of Washington 27: 119-121.

ROBERTS, M S; GITTLEMAN, J L. (1984) Ailurus fulgens. Mammalian Species 222(222): 1-8.

TRAVERSA, D (2011) Are we paying too much attention to cardio-pulmonary nematodes and neglecting old-fashioned worms like Trichuris vulpis? Parasites and Vectors 4: 32.

WANG, X; CHOUDHRY, A; YONGZON, P; WOZEN-CRAFT, C; THAN, Z (2008) Ailurus fulgens. The IUCN Red List of Threatened Species. Version 2015.1. <www.iucnredlist.org>. Downloaded on 18 June 2015.

WEI, F., FENG, Z., WANG, Z. AND HU, J (1999) Current distribution, status and conservation of wild Red pandas Ailurus fulgens in China. Biological conservation 89(3): 285-291.

YONZON, P B; HUNTER, M L. (1991a) Cheese, Tourists and Red pandas in the Nepal Himalayas. Conservation Biology 5(2): 196-202.

YONZON, P B; HUNTER, M L (1991b) Conservation of Red pandas (Ailurus fulgens) Biological Conservation 95: 1-15.

YOSHIMURA, K; HISHINUMA, Y; SATO, M (1969) Ogmocotyle ailuri (Price, 1954) in the Taiwanese monkey, Macaca cyclopis (Swinhoe, 1862). Journal of Parasitology 55: 460-461. 